\title{
Are Diet and Micronutrients Effective in Treating Gastroesophageal Reflux Disease Especially in Women?
}

\author{
Joon Sung Kim and Byung-Wook Kim* \\ Division of Gastroenterology, Department of Internal Medicine, Incheon St. Mary's Hospital, College of Medicine, The Catholic University of \\ Korea, Seoul, Korea
}

\section{Article: Gender-specific effect of micronutrient on non-erosive reflux disease and erosive esophagitis Nam SY, Park BJ, Cho YA, Ryu KH \\ (J Neurogastroenterol Motil 2019;25:82-90)}

Gastroesophageal reflux disease (GERD) is a common disease characterized by reflux of stomach contents into the esophagus. ${ }^{1}$ It is one of the most common gastrointestinal diseases affecting 13$19 \%$ of people worldwide with a greater prevalence reported in the western world. ${ }^{2}$ GERD has been reported to be increasing in Asia over the last 2 decades representing a challenge for physicians in this area. $^{3}$ The costs for treatment of GERD also represent a significant burden on health care systems. ${ }^{4}$

Acid suppression by proton pump inhibitors (PPIs) is considered the primary treatment for GERD. ${ }^{1}$ PPIs are generally regarded to be safe. However, long term use of PPIs have been associated with side effects such as acute and chronic kidney disease, hypomagnesemia, Clostridium difficile infection, and osteoporotic fractures. ${ }^{5}$ Although, the causal relationship between long term PPI use and adverse events remains unproven, these risks underscore the importance of nonpharmacologic management for GERD.

Dietary modification is endorsed by the National Institutes of Health and the American College of Gastroenterology as first-line therapy for patients with GERD. ${ }^{4}$ There exists a paucity of data on the role of diet in the pathogenesis and management of GERD. Coffee, carbonated beverages, alcohol, chocolate, citrus fruits, spicy, acidic, or fried foods have been associated with worsening of reflux symptoms. ${ }^{6}$ Studies have shown an association between food consumption and exacerbation of symptoms. However, this did not necessary correlate with $\mathrm{pH}$ changes. ${ }^{7}$ There is also no convincing evidence that avoidance of these foods can reduce the incidence of GERD symptoms. ${ }^{8}$

In this issue of Journal of Neurogastroenterology and Motility, Nam et al $^{9}$ evaluated the effect of micronutrient intake on erosive esophagitis and non-erosive reflux disease (NERD). This was a cross sectional study including 11690 participants who underwent endoscopy and completed questionnaires for reflux symptoms and dietary intake. The authors aimed to analyze if there was a genderspecific difference in dietary micronutrients on the development of NERD and erosive esophagitis. Prevalence of NERD and erosive esophagitis was $6.8 \%$ and $11.2 \%$ in males and $9.1 \%$ and $2.4 \%$ in females, respectively. High dietary intake of calcium reduced the risk of NERD in men. High dietary intake of vitamin A, retinol, vita-

Received: November 21, 2018 Revised: None Accepted: December 9, 2018

() This is an Open Access article distributed under the terms of the Creative Commons Attribution Non-Commercial License (http://creativecommons. org/licenses/by-nc/4.0) which permits unrestricted non-commercial use, distribution, and reproduction in any medium, provided the original work is properly cited.

${ }^{*}$ Correspondence: Byung-Wook Kim, MD, PhD Division of Gastroenterology, Department of Internal Medicine, College of Medicine, Incheon St. Mary's Hospital, the Catholic University of Korea, 56 Dongsu-ro, Bupyeong-gu, Incheon 21431, Korea Tel: +82-32-280-5052, Fax: +82-2-280-5987, E-mail: gastro@catholic.ac.kr 
min $\mathrm{B} 2$, vitamin $\mathrm{B} 6$, calcium, and iron reduced the risk of NERD in women. Dietary intake of micronutrients was not associated with erosive esophagitis in this study.

The clinical implications of these results remain uncertain. How do micronutrients affect the development of NERD in patients? Why are they different in males and females? What are their association with other known risk factors of GERD such as caffeine, obesity, meal timing and volume? Most importantly, does increasing the intake of micronutrients improve symptoms in NERD patients?

Many foods and beverages have been implicated in exacerbation of GERD symptoms. However, studies supporting that avoidance of foods improves symptoms remain sparse with conflicting results. Some patients may report improvement of symptoms after restriction of certain foods. However, avoidance of specific foods are not routinely recommended in GERD patients as no specific food triggers symptoms in all patients. The authors are applauded for their findings that show a difference in intake of micronutrients in NERD patients. As the authors state in their discussion, the role of micronutrients on development of NERD symptoms is unclear. Due to inherent limitations of a cross sectional study, it is not possible to ascertain a cause and effect relationship between intake of micronutrients and development of NERD.

There is an increasing interest in non-pharmacologic therapeutic options for treatment of GERD. Further research examining the precise roles of dietary control for prevention and treatment of GERD is warranted.
Financial support: None.

Conflicts of interest: None.

\section{References}

1. Vakil N, van Zanten SV, Kahrilas P, Dent J, Jones R; Global Consensus Group. The Montreal definition and classification of gastroesophageal reflux disease: a global evidence-based consensus. Am J Gastroenterol 2006;101:1900-1920.

2. Newberry C, Lynch K. Can we use diet to effectively treat esophageal disease? a review of the current literature. Curr Gastroenterol Rep 2017;19:38.

3. Jung HK. Epidemiology of gastroesophageal reflux disease in Asia: a systematic review. J Neurogastroenterol Motil 2011;17:14-27.

4. Kubo A, Block G, Quesenberry CP Jr, Buffler P, Corley DA. Dietary guideline adherence for gastroesophageal reflux disease. BMC Gastroenterol 2014;14:144.

5. Schoenfeld AJ, Grady D. Adverse effects associated with proton pump inhibitors. JAMA Intern Med 2016;176:172-174.

6. Sethi S, Richter JE. Diet and gastroesophageal reflux disease: role in pathogenesis and management. Curr Opin Gastroenterol 2017;33:107111.

7. Feldman M, Barnett C. Relationships between the acidity and osmolality of popular beverages and reported postprandial heartburn. Gastroenterology 1995;108:125-131.

8. Kaltenbach T, Crockett S, Gerson LB. Are lifestyle measures effective in patients with gastroesophageal reflux disease? An evidence-based approach. Arch Intern Med 2006;166:965-971.

9. Nam SY, Park BJ, Cho YA, Ryu KH. Gender-specific effect of micronutrient on non-erosive reflux disease and erosive esophagitis. J Neurogastroenterol Motil 2019;25:82-90. 